\title{
The Extragalactic Radio Sky at Faint Flux Densities
}

\author{
Carole Jackson $^{\mathrm{A}}$ \\ A CSIRO Australia Telescope National Facility, Epping NSW 1710, Australia. \\ Email: Carole.Jackson@csiro.au
}

Received 2003 January 20, accepted 2004 October 12

\begin{abstract}
In this paper we present simulations of the extragalactic radio sky at 151,325 , and $1400 \mathrm{MHz}$ based on number density predictions from models of the evolution of the radio luminosity function. From these simulations we predict typical source distributions, allowing us to estimate the natural confusion limit to faint flux density limits. The results are relevant to the science and design goals of future radio facilities, namely the Square Kilometre Array, the low-frequency array, and their demonstrator projects.
\end{abstract}

Keywords: radio continuum: galaxies — galaxies: active — quasars: general

\section{Introduction}

Deep continuum observations are a powerful probe of extragalactic sources across a wide range of radio powers and redshifts. The current large-area radio surveys, e.g. the NRAO VLA Sky Survey (NVSS; Condon et al. 1998), Faint Images of the Radio Sky at $20 \mathrm{~cm}$ (FIRST; White et al. 1997), and the Sydney University Molonglo Sky Survey (SUMSS: Bock, Large, \& Sadler 1999), cover thousands of square degrees of sky to mJy flux density limits. These surveys are the deepest practicable over large areas of sky given the large amounts of observing time involved. Surveys to increasingly fainter flux density limits cover far smaller areas, e.g. the Australia Telescope ESO Slice Project (ATESP; Prandoni et al. 2000) covers $26 \mathrm{deg}^{2}$ to $S_{1400 \mathrm{MHz}}=0.4 \mathrm{mJy}(5 \sigma \mathrm{rms})$, whereas the VLA observations of the Hubble Deep Field North area cover just $0.35 \mathrm{deg}^{2}$ (Richards 2000) to $S_{1400 \mathrm{MHz}}=0.04 \mathrm{mJy}$ $(5 \sigma \mathrm{rms})$. The next generation of radio telescopes, with their significantly larger collecting areas, will be capable of large-area sub- $\mu$ Jy surveys. The challenge is to determine what the sky might look like at these faint limits.

At bright flux densities (i.e. $>1 \mathrm{Jy}$ at all frequencies), continuum radio surveys are dominated by radio-loud galaxies and quasars, collectively termed as radio-loud active galactic nuclei (AGN). It is only at mJy flux densities that any other population forms a sizeable contribution to the total source count. Whilst there are only incomplete, or local, spectroscopic samples at sub-mJy flux densities, all identify a significant contribution from late-type ('star-forming') galaxies (ATESP: Prandoni et al. 2001; 2dFGRS-NVSS: Sadler et al. 2002; 2dFGRS-FIRST: Magliocchetti et al. 2002).

Since the radio waveband is unaffected by dust obscuration it provides an unbiased probe of both the AGN and star-forming galaxy phenomena. It is yet to be established whether the AGN detected at faint (sub-mJy) flux densities are higher-redshift and/or lower-power counterparts of those detected in bright surveys. Thus future ultra-deep radio continuum surveys promise to probe at least three key astrophysical questions: (a) the starformation history of the Universe, (b) the evolution of the low-power end of the radio galaxy luminosity function, and (c) any relationship or interplay between AGN, star formation, and the radio-quiet AGN phenomenon.

In this paper we present sky simulations at 151,325 , and $1400 \mathrm{MHz}$ giving the expected sky density and redshift distribution of extragalactic radio sources based on our models of the evolution of the radio luminosity functions for the 'parent populations' of Fanaroff-Riley class I and II (FRI and FRII) radio galaxies (Fanaroff \& Riley 1972). We incorporate the contribution from the star-forming galaxies, using the locally derived radio luminosity function and an evolution model from the deepest radio surveys to date (Section 4.1). Our approach is in contrast to the earlier work of Hopkins et al. (2000) where the AGN sources were accounted for using the evolving radio luminosity functions for the 'flat' and 'steep' sources, as determined by Dunlop \& Peacock (1990), and the optical luminosity function coupled with an assumed, constant radio-optical luminosity ratio to incorporate the contribution from star-forming galaxies.

We assume a characteristic size distribution for each class of object. As a result we can deduce a natural confusion limit, occurring when the surface density of objects is so high that one has less than some minimum number of beam areas per detected object.

In this paper we adopt $H_{0}=50 \mathrm{~km} \mathrm{~s}^{-1} \mathrm{Mpc}^{-1}$ and $q_{0}=0.5 \quad\left(\Omega_{\mathrm{m}}=1\right)$ cosmology. We also show the corresponding results for the WMAP (lambdadominated) cosmology with $\Omega_{\mathrm{m}}=0.27, \Omega_{\lambda}=0.73$, and $H_{0}=71 \mathrm{~km} \mathrm{~s}^{-1} \mathrm{Mpc}^{-1}$. However, detailed discussion and interpretation of the latter case is reserved for a subsequent paper as it requires a WMAP cosmology-based solution 
Table 1. Target specifications for SKAMP

\begin{tabular}{|c|c|c|c|}
\hline Parameter & $300 \mathrm{MHz}$ & $843 \mathrm{MHz}$ & $1420 \mathrm{MHz}$ \\
\hline Resolution $\left(\delta<-30^{\circ}\right)$ & $123^{\prime \prime} \times 123^{\prime \prime} \csc \delta$ & $45^{\prime \prime} \times 45^{\prime \prime} \csc \delta$ & $26^{\prime \prime} \times 26^{\prime \prime} \csc \delta$ \\
\hline Imaging FOV & $7.7^{\circ} \times 7.7^{\circ} \csc \delta$ & $2.5^{\circ} \times 2.5^{\circ} \csc \delta$ & $1.5^{\circ} \times 1.5^{\circ} \csc \delta$ \\
\hline UV coverage & Fully sampled & Fully sampled & Fully sampled \\
\hline$T_{\text {sys }}$ & $<150 \mathrm{~K}$ & $<80 \mathrm{~K}$ & $<50 \mathrm{~K}$ \\
\hline System noise $(1 \sigma) 12 \mathrm{~h}$ & $33 \mu \mathrm{Jy}_{\text {beam }}{ }^{-1}$ & $18 \mu \mathrm{Jy}_{\text {beam }^{-1}}$ & $11 \mu \mathrm{Jy}_{\text {beam }}{ }^{-1}$ \\
\hline $\begin{array}{l}12 \mathrm{~h}, 5 \sigma \text { point-source } \\
\text { sensitivity }^{\mathrm{A}}\end{array}$ & $2.0 \mathrm{mJy}$ & $1.0 \mathrm{mJy}$ & $0.2 \mathrm{mJy}$ \\
\hline
\end{tabular}

A Indicative values provided by J. D. Bunton (2002, private communication).

for the evolution of the star-forming galaxy population which is not yet determined: the star-forming galaxy sample from the AT Hubble Deep Field South sample at $20 \mathrm{~cm}$ should provide this model (M. T. Huynh et al., in preparation). Therefore our results should be treated as a first draft, given the approximation of the star-forming galaxy evolution which we adopted to account for the larger differential volumes in the lambda-dominated cosmology.

\section{Future Radio Facilities}

Two future radio telescopes, the low-frequency array (LOFAR; www.lofar.org) and the Square Kilometre Array (SKA; www.skatelescope.org/), are proposed with huge sensitivity improvements at sub$\mathrm{GHz}$ frequencies compared to current-day telescopes. LOFAR has a specified frequency range between $10 \mathrm{MHz}$ and $240 \mathrm{MHz}$. The SKA operating frequencies are not finalised, but are likely to extend from around $100 \mathrm{MHz}$ to $20 \mathrm{GHz}$.

As pathways to the SKA, a number of new facilities will be developed as 'new technology demonstrators'. These will be in operation over the next few years. One such project is a three-phase upgrade of the Molonglo Observatory Synthesis Telescope (MOST), project 'SKAMP' (www.physics . usyd. edu . au / astrop/ ska). Phases 1 and 2 will add a continuum correlator at $30 \mathrm{MHz}$ bandwidth. Phase 3 will extend the operating frequency range from 300 to $1420 \mathrm{MHz}$. Thus the fully upgraded facility will be a powerful low-frequency telescope with a wide instantaneous field of view and complete $u v$ coverage inherent to a $1.6-\mathrm{km}$ east-west cylindrical paraboloid telescope. A key feature will be the demonstration of 'multibeaming' via a small number of independent fanbeams.

Table 1 shows the target specifications for the MOST demonstrator SKAMP. The key extragalactic science goal for this SKA demonstrator is to trace $\mathrm{HI}$ in absorption against bright continuum sources over a wide redshift range, where $\lambda_{\mathrm{HI}, \text { rest }}=1420 \mathrm{MHz}$ corresponds to a maximum redshift of 3.7 at $300 \mathrm{MHz}$. The surface density of background radio sources on the sky is the key factor determining whether this will be feasible with this facility. At the end of this paper we discuss the expected surface density of these background sources.

\section{Extragalactic Radio Sources at 151-1400 MHz}

At observing frequencies between $151 \mathrm{MHz}$ and $1400 \mathrm{MHz}$, powerful radio sources are a mixture of radioloud galaxies, quasars, and BL Lac objects - all members of the AGN class of objects. At the zeroth level, radio galaxies can be classified as either FRI or FRII: these are distinguished by the distribution of bright knots of radio emission and collimation features (Fanaroff \& Riley 1972). In general, FRIs are of lower radio power than FRIIs, although there is considerable overlap in the radio luminosity function of the two classes. Quasars and BL Lac objects are manifestations of FRI and FRII radio galaxies aligned close to our line of sight, with their core radio emission Doppler-beamed (Jackson \& Wall 1999, and references therein).

In this paper we base our sky simulations on data at low frequency where the source count is well determined and the effects of Doppler beaming are negligible. From $151 \mathrm{MHz}$ source count data and the observed luminosity distribution of the 3CRR catalogue (Laing, Riley, \& Longair 1983), we derive the evolution of the FRI and FRII populations. As we are extrapolating to sub-mJy flux densities we include the contribution from star-forming galaxies which are generally of lower radio power than FRIs and FRIIs and whose radio synchrotron emission originates from supernovae rather than AGN. This category of star formers is dominated by late-type galaxies with a wide range of ongoing star-formation rates - from the extreme Arp220-like galaxies to normal galaxies like our own Milky Way.

The prediction of the radio sky at frequencies below $151 \mathrm{MHz}$ is outside the scope of this paper.

\section{Predicting the Radio Sky}

\subsection{Radio Source Evolution}

If we assume that the radio sky comprises three populations of galaxies, namely FRI, FRII, and star-forming, we can determine the nature of the radio sky, i.e. surface density, redshift distribution, and luminosities, at a range of frequencies if we know the local radio luminosity function (LRLF) and its evolution for each population.

For the star-forming galaxy population we use the LRLF determined by Sadler et al. (2002) from the 
2dFGRS-NVSS galaxy sample at $1400 \mathrm{MHz}$. For the models at $151 \mathrm{MHz}$ and $325 \mathrm{MHz}$ we transpose the starforming galaxy LRLF using a single-value spectral index. We adopt a spectral index of -0.7 in accord with that determined by Condon (1983) for spiral galaxies at low frequencies. At present it is not clear if this value is generally applicable to this class given the scant amount of low-frequency data available: in particular bright star-forming galaxies exhibit low-frequency spectral flattening due to free-free absorption in their HII regions (Condon 1992) and subsequently have a spectral index around -0.4 . The consequence of using an index of -0.7 rather than a flatter value for the whole population is that the contribution from star-formers is overpredicted in the sky simulations at 151 and $325 \mathrm{MHz}$. We adopt parameterised number-density luminosity evolution for the star-forming population as determined from the HDF-N and SSA13 fields based on $\Omega_{\mathrm{m}}=1$ cosmology (Haarsma et al. 2000).

To determine the LRLFs and evolution for the FRI and FRII radio galaxies we adopt the methodology of Jackson \& Wall (1999). In summary, we fit exponential luminosity-dependent density evolution to the observed source count at $151 \mathrm{MHz}$. The count at $151 \mathrm{MHz}$ comprises data from the 3CRR catalogue (Laing et al. 1983) and the 6C survey (Hales, Baldwin, \& Warner 1988). We use the spectral curvature fits of Jackson \& Wall (2001) to describe the spectral shape of radio galaxies which deviates from the 'canonical value' of $\alpha=-0.7$ for $S \propto \nu^{\alpha}$.

We find a best fit to the observed $151 \mathrm{MHz}$ source count for the case where there is zero evolution of the FRI population and luminosity-dependent density evolution of the FRII population, $\phi_{z}=F(P, z) \cdot \phi_{0}$, where $\phi_{0}$ is the LRLF. For the function $F(P, z)$ we fit $M_{\max }=15.23, z_{\mathrm{c}}=4.20$, $P_{1}=25.55$, and $P_{2}=27.76$ (equation 3 in Jackson \& Wall 1999). These parameter values have a chi-squared value of 29.7 for 33 degrees of freedom for $\Omega_{\mathrm{m}}=1$ cosmology. The observed and model differential source counts are shown in Figure 1. Figure 2 shows the resultant LRLF at $151 \mathrm{MHz}$ comprising contributions from the three radio source populations.

Applying the same evolution functions to luminosity functions based on WMAP cosmology, we find the best fit at $M_{\max }=15.70, z_{\mathrm{c}}=7.425, P_{1}=25.94$, and $P_{2}=27.94$ for the FRII population, this time coupled with mild $(M=-3.06)$ density evolution of the FRI population. This fit has a chi-squared value of 30.2. As there is no WMAP cosmology determination of the star-forming evolution we can adopt the fit determined by Haarsma et al. (2000) and normalise the number of star-forming galaxies to produce a fit to the source count.

Our procedure assumes that we have accounted for all constituent populations at the frequencies under consideration. There is the possibility that we are excluding particular classes of source which are not detected in the $151 \mathrm{MHz}$ source count. For example, above $400 \mathrm{MHz}$ there is a significant population of gigahertz peaked spectrum (GPS) and compact steep spectrum (CSS) sources.

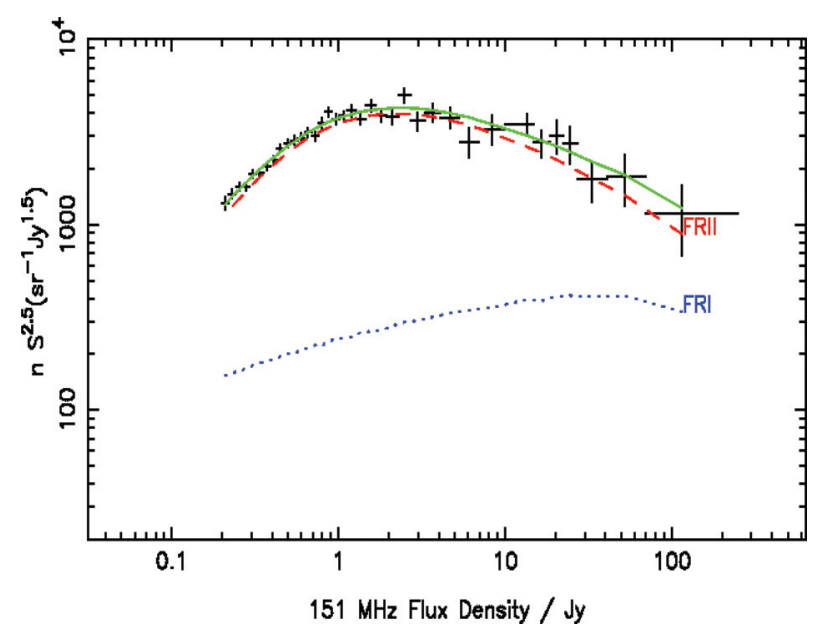

Figure 1 Normalised observed and model differential source counts at $151 \mathrm{MHz}$ for $\Omega_{\mathrm{m}}=1$ cosmology. The model is the best fit to the observed $151 \mathrm{MHz}$ source count as described in Section 3.1 using data points $(+)$ from the $3 \mathrm{CR}$ count from the 3CRR catalogue at $178 \mathrm{MHz}$ (Laing et al. 1983) and the 6C survey (Hales et al. 1988). The model fit comprises contributions from two populations described in the text: FRII radio galaxies (dashed line) and FRI radio galaxies (dotted line).

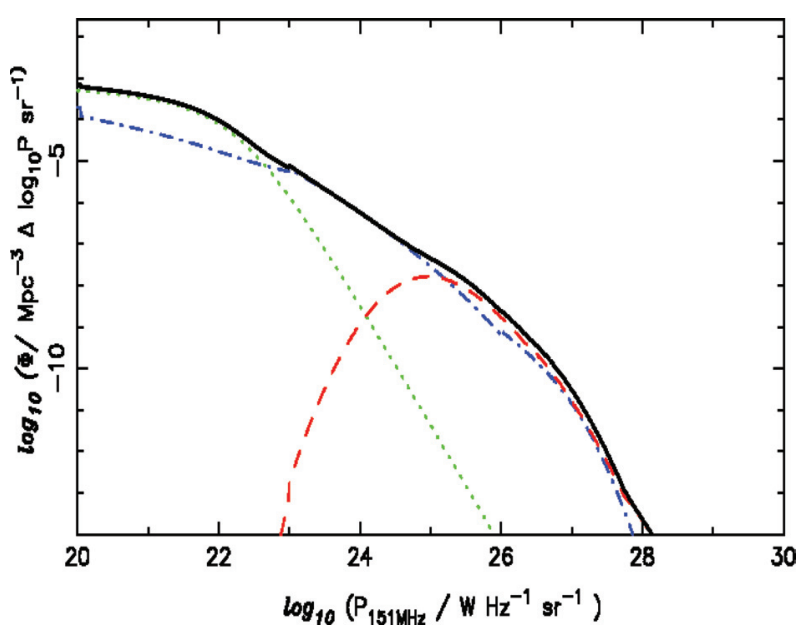

Figure 2 The LRLF at $151 \mathrm{MHz}$ derived from the best-fit model $\left(\Omega_{\mathrm{m}}=1\right)$ to the observed source count at $151 \mathrm{MHz}$ (solid line). The LRLF comprises contributions from three populations: starforming galaxies (dotted), FRI radio sources (dot-dash), and FRII radio sources (dashed).

At present it is unclear how these sources relate to the FRI and FRII radio galaxy populations. They form a significant fraction $(\sim 15 \%)$ of all 3C sources (Fanti et al. 1990) and as such they are implicitly incorporated into our analysis, with the assumption that they follow the same evolution as all other ('normal') FRII radio galaxies.

\subsection{Translating a Differential Source Count to a Model Sky}

With the local luminosity functions and evolution factors derived in the previous section we produce simulated sky regions at $151 \mathrm{MHz}$. For each single simulation we randomly position each source within a specified sky 


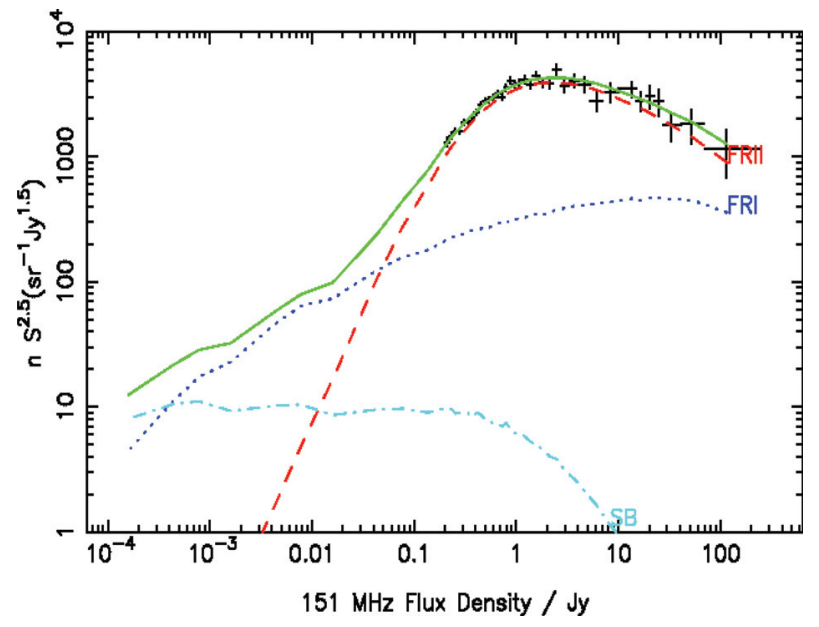

Figure 3 Differential source count at $151 \mathrm{MHz}$ for $\Omega_{\mathrm{m}}=1$ cosmology, with the model extrapolated beyond the observed count using the evolving LRLF as described in the text. Observed data points $(+)$ and model contributions are as for Figure 1 with the star-forming galaxies (dot-dash) making a significant contribution below $1 \mathrm{mJy}$.

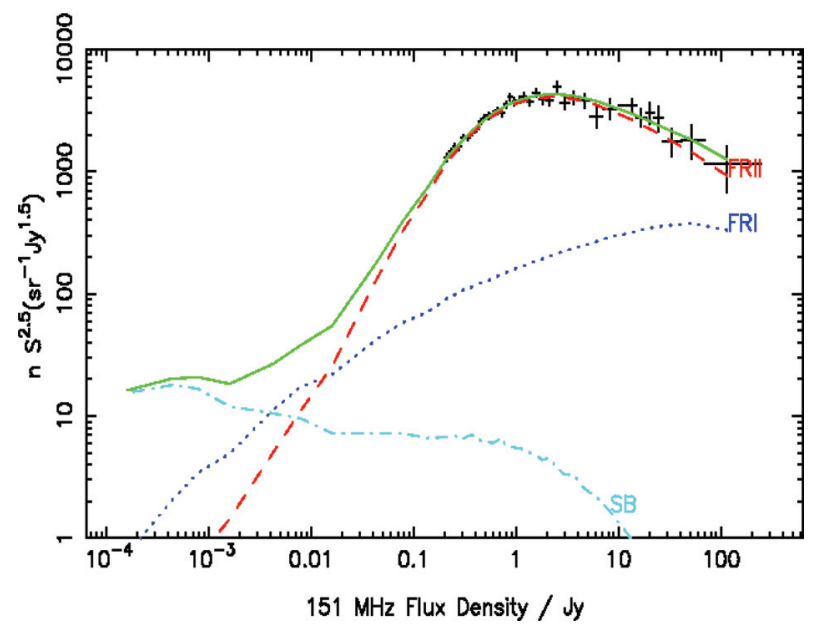

Figure 4 Differential source count at $151 \mathrm{MHz}$ for the lambdadominated cosmology with the model extrapolated beyond the observed count using the evolving LRLF as described in the text. Observed data points $(+)$ and model contributions are as for Figure 1 with the star-forming galaxies (dot-dash) making a significant contribution below $1 \mathrm{mJy}$. Compared to the $\Omega_{\mathrm{m}}=1 \mathrm{fit}$, the FRII population extends to higher redshifts and is the dominant source population above $\sim 20 \mathrm{mJy}$.

Table 2. Predicted source surface densities at $151 \mathrm{MHz}\left(\Omega_{\mathrm{m}}=1\right)$

\begin{tabular}{|c|c|c|c|c|c|c|c|c|c|c|}
\hline \multirow[t]{3}{*}{ Population } & \multicolumn{10}{|c|}{ Flux density limit } \\
\hline & \multicolumn{2}{|c|}{$10 \mathrm{mJy}$} & \multicolumn{2}{|c|}{$5 \mathrm{mJy}$} & \multicolumn{2}{|c|}{$2 \mathrm{mJy}$} & \multicolumn{2}{|c|}{$1 \mathrm{mJy}$} & \multicolumn{2}{|c|}{$100 \mu \mathrm{Jy}$} \\
\hline & $\begin{array}{c}N \\
{\left[\mathrm{deg}^{-2}\right]}\end{array}$ & $\begin{array}{l}\text { Cover } \\
\text { fraction }\end{array}$ & $\begin{array}{c}N \\
{\left[\mathrm{deg}^{-2}\right]}\end{array}$ & $\begin{array}{l}\text { Cover } \\
\text { fraction }\end{array}$ & $\begin{array}{c}N \\
{\left[\mathrm{deg}^{-2}\right]}\end{array}$ & $\begin{array}{l}\text { Cover } \\
\text { fraction }\end{array}$ & $\begin{array}{c}N \\
{\left[\mathrm{deg}^{-2}\right]}\end{array}$ & $\begin{array}{l}\text { Cover } \\
\text { fraction }\end{array}$ & $\begin{array}{c}N \\
{\left[\mathrm{deg}^{-2}\right]}\end{array}$ & $\begin{array}{l}\text { Cover } \\
\text { fraction }\end{array}$ \\
\hline FRI & 12 & $1 \times 10^{-3}$ & 31 & $3 \times 10^{-3}$ & 76 & $6 \times 10^{-3}$ & 116 & $9 \times 10^{-3}$ & 744 & $4 \times 10^{-2}$ \\
\hline FRII & 16 & $5 \times 10^{-4}$ & 19 & $7 \times 10^{-4}$ & 20 & $7 \times 10^{-4}$ & 21 & $7 \times 10^{-4}$ & 23 & $8 \times 10^{-4}$ \\
\hline Star-forming & 2 & $6 \times 10^{-6}$ & 8 & $2 \times 10^{-5}$ & 35 & $6 \times 10^{-5}$ & 72 & $1 \times 10^{-4}$ & 2046 & $1 \times 10^{-3}$ \\
\hline Total & 30 & $2 \times 10^{-3}$ & 58 & $4 \times 10^{-3}$ & 131 & $7 \times 10^{-3}$ & 209 & $1 \times 10^{-2}$ & 2813 & $4 \times 10^{-2}$ \\
\hline Minimum resolution & \multicolumn{2}{|c|}{$22^{\prime \prime}$} & \multicolumn{2}{|c|}{$15^{\prime \prime}$} & \multicolumn{2}{|c|}{$9^{\prime \prime}$} & \multicolumn{2}{|c|}{$7^{\prime \prime}$} & \multicolumn{2}{|c|}{$1^{\prime \prime}$} \\
\hline Sources overlapped & \multicolumn{2}{|c|}{$0.9 \%$} & \multicolumn{2}{|c|}{$1.7 \%$} & \multicolumn{2}{|c|}{$2.4 \%$} & \multicolumn{2}{|c|}{$3.5 \%$} & \multicolumn{2}{|c|}{$11.5 \%$} \\
\hline Sky confused & \multicolumn{2}{|c|}{$0 \%$} & \multicolumn{2}{|c|}{$0 \%$} & \multicolumn{2}{|c|}{$0 \%$} & \multicolumn{2}{|c|}{$0.001 \%$} & \multicolumn{2}{|c|}{$0.031 \%$} \\
\hline
\end{tabular}

Table 3. Predicted source surface densities at $151 \mathrm{MHz}$ (lambda-dominated)

\begin{tabular}{|c|c|c|c|c|c|c|c|c|c|c|}
\hline \multirow[t]{3}{*}{ Population } & \multicolumn{10}{|c|}{ Flux density limit } \\
\hline & \multicolumn{2}{|c|}{$10 \mathrm{mJy}$} & \multicolumn{2}{|c|}{$5 \mathrm{mJy}$} & \multicolumn{2}{|c|}{$2 \mathrm{mJy}$} & \multicolumn{2}{|c|}{$1 \mathrm{mJy}$} & \multicolumn{2}{|c|}{$100 \mu \mathrm{Jy}$} \\
\hline & $\begin{array}{c}N \\
{\left[\mathrm{deg}^{-2}\right]}\end{array}$ & $\begin{array}{l}\text { Cover } \\
\text { fraction }\end{array}$ & $\begin{array}{c}N \\
{\left[\mathrm{deg}^{-2}\right]}\end{array}$ & $\begin{array}{l}\text { Cover } \\
\text { fraction }\end{array}$ & $\begin{array}{c}N \\
{\left[\mathrm{deg}^{-2}\right]}\end{array}$ & $\begin{array}{l}\text { Cover } \\
\text { fraction }\end{array}$ & $\begin{array}{c}N \\
{\left[\mathrm{deg}^{-2}\right]}\end{array}$ & $\begin{array}{l}\text { Cover } \\
\text { fraction }\end{array}$ & $\begin{array}{c}N \\
{\left[\mathrm{deg}^{-2}\right]}\end{array}$ & $\begin{array}{l}\text { Cover } \\
\text { fraction }\end{array}$ \\
\hline FRI & 6 & $1 \times 10^{-3}$ & 15 & $3 \times 10^{-3}$ & 34 & $4 \times 10^{-3}$ & 50 & $6 \times 10^{-3}$ & 275 & $6 \times 10^{-2}$ \\
\hline FRII & 18 & $7 \times 10^{-4}$ & 23 & $9 \times 10^{-4}$ & 29 & $1 \times 10^{-3}$ & 33 & $1 \times 10^{-3}$ & 52 & $6 \times 10^{-4}$ \\
\hline Star-forming & 1 & $5 \times 10^{-6}$ & 8 & $2 \times 10^{-5}$ & 44 & $7 \times 10^{-5}$ & 89 & $1 \times 10^{-4}$ & 3810 & $8 \times 10^{-4}$ \\
\hline Total & 25 & $2 \times 10^{-3}$ & 46 & $4 \times 10^{-3}$ & 107 & $6 \times 10^{-3}$ & 172 & $8 \times 10^{-3}$ & 4137 & $6 \times 10^{-2}$ \\
\hline Minimum resolution & \multicolumn{2}{|c|}{$25^{\prime \prime}$} & \multicolumn{2}{|c|}{$17^{\prime \prime}$} & \multicolumn{2}{|c|}{$11^{\prime \prime}$} & \multicolumn{2}{|c|}{$8^{\prime \prime}$} & \multicolumn{2}{|c|}{$1.5^{\prime \prime}$} \\
\hline Sources overlapped & \multirow{2}{*}{\multicolumn{2}{|c|}{$0.8 \%$}} & \multirow{2}{*}{\multicolumn{2}{|c|}{$\begin{array}{c}1.4 \% \\
0 \%\end{array}$}} & \multirow{2}{*}{\multicolumn{2}{|c|}{$1.9 \%$}} & \multicolumn{2}{|c|}{$2.4 \%$} & \multicolumn{2}{|c|}{$16.3 \%$} \\
\hline Sky confused & & & & & & & \multicolumn{2}{|c|}{$0.001 \%$} & \multicolumn{2}{|c|}{$0.004 \%$} \\
\hline
\end{tabular}

area. Each source is then randomly oriented, both on and into the plane. FRI and FRII radio galaxies are randomly assigned a total intrinsic size (lobe-to-lobe) between 50 and $800 \mathrm{kpc}$ assuming no source size evolution with redshift. These source sizes are in line with radio sources studied in the literature, e.g. the 3CRR sample (Laing et al. 1983). FRIs and FRIIs are modelled as doublelobe structures, with each lobe being half the intrinsic 
total size adjusted for source orientation and redshift, i.e. foreshortened and flattened.

Each star-forming galaxy is modelled as a circular disk galaxy with a randomly assigned intrinsic disk diameter between 10 and $100 \mathrm{kpc}$. Given the increasing evidence that spiral disks are significantly smaller than $10 \mathrm{kpc}$ at high redshift (Giallongo et al. 2000), our adopted range is almost certainly too large, and the sky coverage due to star-forming galaxies will be overstated. However, as we are interested in a 'conservative' prediction of source density and confusion rates, we deliberately work with this extreme set of sizes. The galaxy disks are oriented randomly on the plane of the sky.

\subsection{The Radio Sky at $151 \mathrm{MHz}$}

Using the local luminosity functions and evolution factors derived in Section 4.1, we calculate the differential source count down to $100 \mu \mathrm{Jy}$ for each of the two cosmologies (Figures 3 and 4).

From the differential source count we can extract the expected number of sources per square degree and the fraction of sky covered by each population to any flux density limit. Statistics for a range of flux densities at $151 \mathrm{MHz}$ are given in Tables 2 and 3. Simulated sky images at $151 \mathrm{MHz}$ for these flux density limits are shown in Appendix A.

By averaging the results of 1000 sky simulations we determine the minimum resolution which would give an average intersource spacing of 10 primary beams on the sky, given the varying set of source sizes in each single sky simulation. Between $S_{151 \mathrm{MHz}}=10 \mathrm{mJy}$ and $100 \mu \mathrm{Jy}$ the minimum resolution requirement to avoid natural confusion ranges from 22 to $1 \operatorname{arcsec}\left(\Omega_{\mathrm{m}}=1\right)$. As shown in Tables 2 and 3 the source sky densities are similar: the two cosmologies differ in their differential volumes (and hence redshift distributions), whereas the source surface densities must be constant to match the observed radio source count.

We also calculate the proportion of sources which overlap each other on the plane of the sky. We find that this varies from $0.9 \%$ of all sources at $10 \mathrm{mJy}$, rising to $11.5 \%$ at $100 \mu \mathrm{Jy}$. This means that at a flux density limit of $S_{151 \mathrm{MHz}}=100 \mu \mathrm{Jy}$, one in nine sources will be in line-ofsight coincidence with another source for an instrument with 1 arcsec resolution or better. Even at this low flux density limit the fraction of sky area which exhibits this effect is very small, being approximately one hundredth of the total area covered by sources.

Figures 5 and 6 show the expected redshift distributions for the one square degree sky areas for flux density limits of 5, 2, and $1 \mathrm{mJy}$ for the two cosmologies. In both there is a peak in $N(z)$ at low redshift $(z \sim 0.1)$ due to the high space density of star-forming galaxies at low radio powers and low redshifts. Beyond $z \sim 2.5$ for $\Omega_{\mathrm{m}}=1$, and $z \sim 4$ for lambda-dominated cosmology, $N(z)$ drops rapidly.

Figures 7, 8, and 9 illustrate the luminosity-redshift distributions for flux-limited samples at $1 \mathrm{mJy}$ and

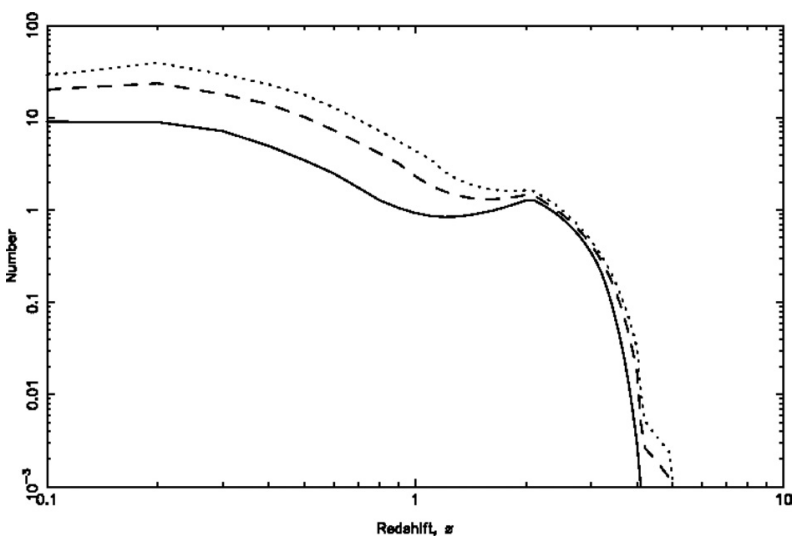

Figure 5 Predicted redshift distribution, $N(z)$, at $151 \mathrm{MHz}$ for $\Omega_{\mathrm{m}}=1$ cosmology. Number of sources per $0.1 \Delta z$, per square degree of sky for flux density limits of $5 \mathrm{mJy}$ (solid line), $2 \mathrm{mJy}$ (dashed line), and $1 \mathrm{mJy}$ (dotted line) is derived from the fit shown in Figure 3.

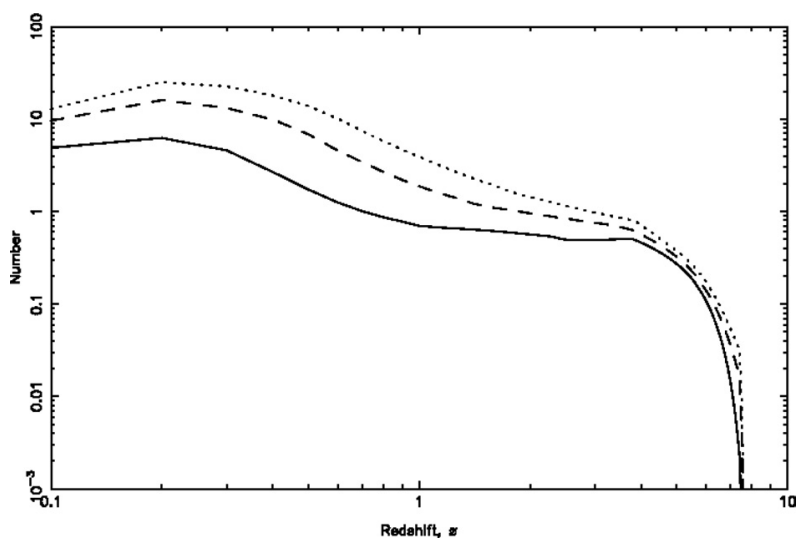

Figure 6 Predicted redshift distribution, $N(z)$, at $151 \mathrm{MHz}$ for the lambda-dominated cosmology. Number of sources per $0.1 \Delta z$ per square degree of sky for flux density limits of $5 \mathrm{mJy}$ (solid line), $2 \mathrm{mJy}$ (dashed line), and $1 \mathrm{mJy}$ (dotted line) is derived from the fit shown in Figure 4.

$100 \mu \mathrm{Jy}$ for the $\Omega_{\mathrm{m}}=1$ cosmology. At $1 \mathrm{mJy}$ the highredshift tail is comprised of FRII radio sources, whereas FRI and star-forming sources lie at $z<1.5$. At $100 \mu \mathrm{Jy}$ all three populations extend to high redshift $(z \sim 4)$. Figures 10,11 , and 12 illustrate the luminosity-redshift distributions for flux-density limited samples at $1 \mathrm{mJy}$ and $100 \mu \mathrm{Jy}$ in the case of the lambda-dominated cosmology. Compared to the $\Omega_{\mathrm{m}}=1$ predictions, the FRII radio galaxies extend to higher redshift in the lambda-dominated model fit.

\subsection{The Radio Sky at $325 \mathrm{MHz}$}

We calculate the source count at $325 \mathrm{MHz}$ to a flux density limit of $100 \mu \mathrm{Jy}$ based on the evolving LRLF derived at $151 \mathrm{MHz}$. We apply spectral curvature fits (Jackson \& Wall 2001) to the rest-frame radio emission for the FRI and FRII populations and for the star-forming galaxies we use a standard spectral index of -0.7 to shift the LRLF to $325 \mathrm{MHz}$. At bright flux density limits $\left(S_{325 \mathrm{MHz}}>50 \mathrm{mJy}\right)$, the model differential count closely 


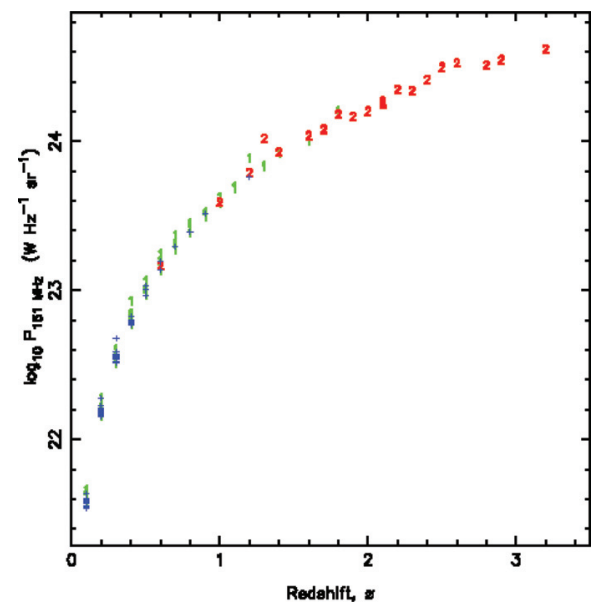

Figure 7 Power-redshift distribution, $P-z$, at $151 \mathrm{MHz}$ for a flux density limit of $1 \mathrm{mJy}$ derived from the fit shown in Figure $3\left(\Omega_{\mathrm{m}}=1\right)$. The star-forming galaxies are confined to redshifts below $\sim 1$. Key: FRI radio galaxies (1), FRII radio galaxies (2), star-forming galaxies $(+)$.

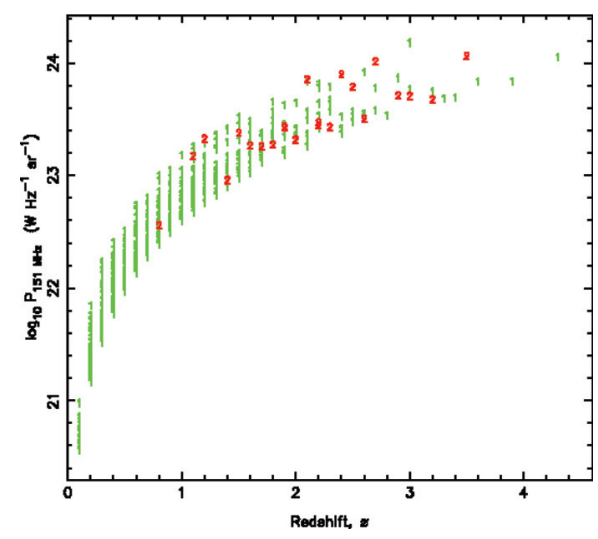

Figure 8 Power-redshift distribution, $P-z$, at $151 \mathrm{MHz}$ for a flux density limit of $100 \mu \mathrm{Jy}$ derived from the fit shown in Figure 3 $\left(\Omega_{\mathrm{m}}=1\right)$. At each redshift interval $(\Delta z=0.1)$ the vertical spread in radio powers is set by the breadth of the radio luminosity function at this redshift. Key: FRI radio galaxies (1), FRII radio galaxies (2).

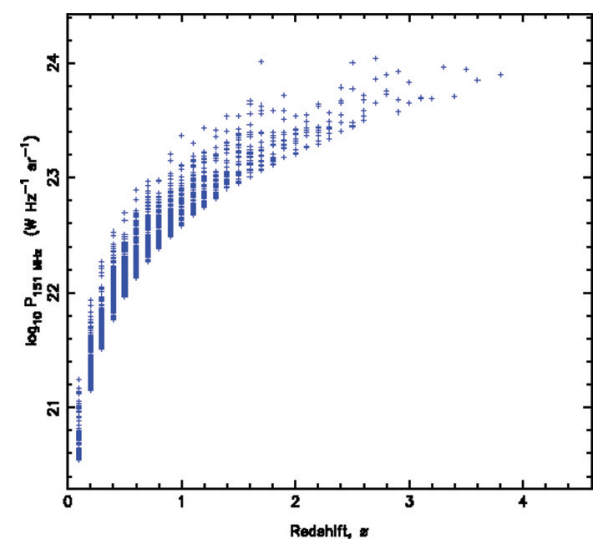

Figure 9 Power-redshift distribution, $P-z$, for star-forming galaxies $(+)$ at $151 \mathrm{MHz}$ for a flux density limit of $1 \mathrm{mJy}$ derived from the fit shown in Figure $3\left(\Omega_{\mathrm{m}}=1\right)$. At this flux density the starforming galaxies dominate and extend to $z \sim 4$. The abrupt cut-off at $z=3.97$ is set by the model fit of Haarsma et al. (2000). At each redshift interval $(\Delta z=0.1)$ the vertical spread in radio powers is set by the breadth of the radio luminosity function at this $z$.

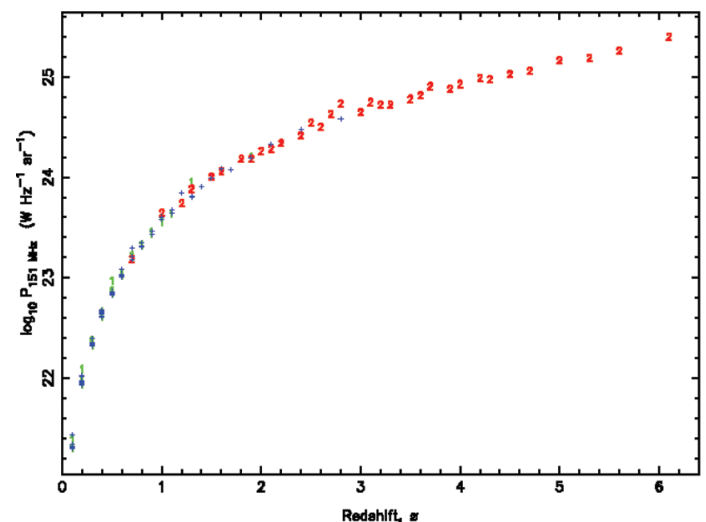

Figure 10 Power-redshift distribution, $P-z$, at $151 \mathrm{MHz}$ for a flux density limit of $1 \mathrm{mJy}$ derived from the fit shown in Figure 4 (lambda-dominated cosmology). Compared to the $\Omega_{\mathrm{m}}=1 \mathrm{fit}$, FRII galaxies dominate beyond $z \sim 1$ and extend to high redshift $(z \sim 6)$. Key: FRI radio galaxies (1), FRII radio galaxies (2), star-forming galaxies $(+)$.

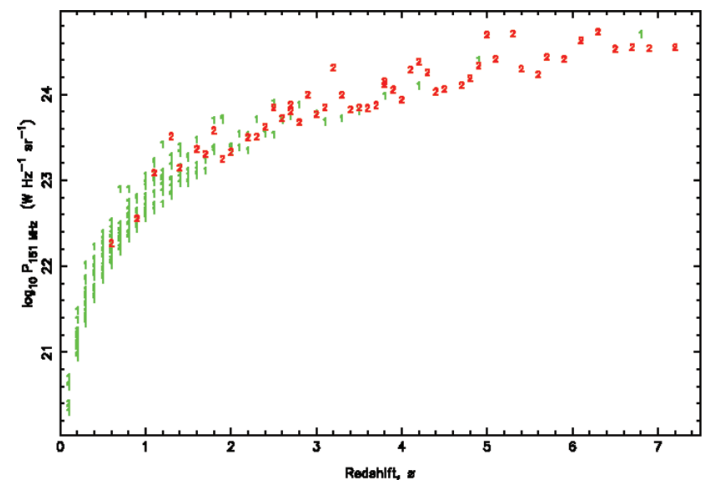

Figure 11 Power-redshift distribution, $P-z$, for FRI and FRII sources at $151 \mathrm{MHz}$ for a flux density limit of $100 \mu \mathrm{Jy}$ derived from the fit shown in Figure 4 (lambda-dominated cosmology). The FRI sources dominate above $z \sim 3$ and extend to high redshift. At each redshift interval $(\Delta z=0.1)$ the vertical spread in radio powers is set by the breadth of the radio luminosity function at this redshift. Key: FRI radio galaxies (1), FRII radio galaxies (2).

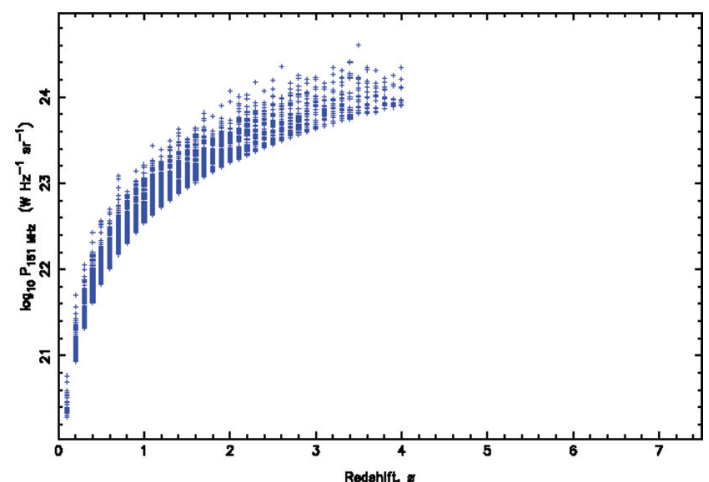

Figure 12 Power-redshift distributions, $P-z$, for star-forming galaxies (+) at $151 \mathrm{MHz}$ for a flux density limit of $100 \mu \mathrm{Jy}$ derived from the fit shown in Figure 4 (lambda-dominated cosmology). At each redshift interval $(\Delta z=0.1)$ the vertical spread in radio powers is set by the breadth of the radio luminosity function at this redshift. 
matches that of the Westerbork Northern Sky Survey (WENSS; Rengelink et al. 1997), as shown in Figure 13.

The number of sources per square degree and the coverage fraction for each population at $325 \mathrm{MHz}$ are given in Table 4 . We find that the total sky coverage fraction at the lowest flux density limit $(100 \mu \mathrm{Jy})$ is 0.03 , i.e. that there is very limited overlapping of sources on the sky.

From 1000 unique sky simulations we determine the minimum resolution which would yield an average intersource spacing of 10 primary beams on the sky. Between $S_{325 \mathrm{MHz}}=5 \mathrm{mJy}$ and $100 \mu \mathrm{Jy}$ we find that the minimum resolution requirement to avoid natural confusion ranges from 19 to 2 arcsec. The results for the lambda-dominated cosmology are very similar for the reasons discussed in Section 4.3.

Figure 14 shows the expected redshift distribution for the one square degree sky areas for flux density limits of 2,1 , and $0.1 \mathrm{mJy}$ for $\Omega_{\mathrm{m}}=1$ cosmology. As at $151 \mathrm{MHz}$, beyond the peak space density at $z=2.5 N(z)$

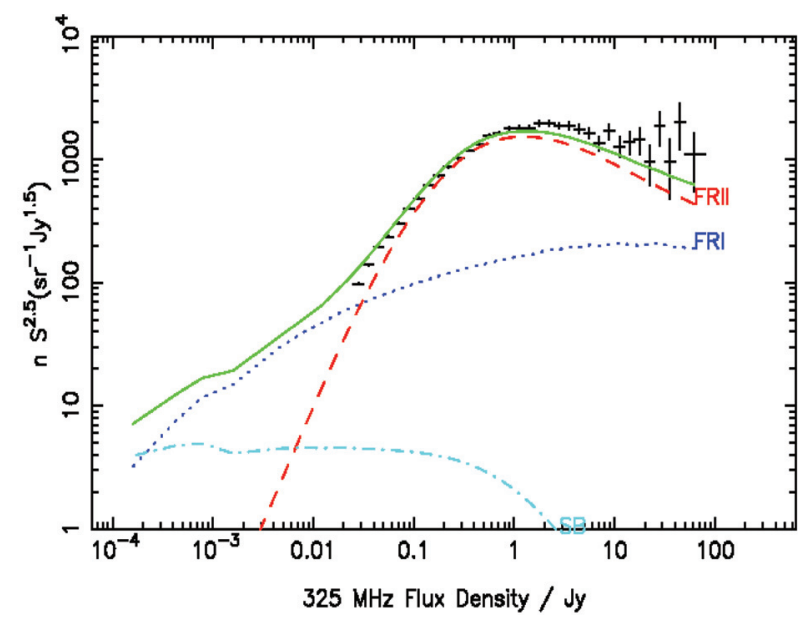

Figure 13 Normalised observed and model differential source counts at $325 \mathrm{MHz}\left(\Omega_{\mathrm{m}}=1\right)$. The model count is derived from the evolving LRLF at $151 \mathrm{MHz}$ as described in the text and is comprised of three populations: FRII radio galaxies (dashed line), FRI radio galaxies (dotted line), and star-forming galaxies (dot-dash line). Data points $(+)$ are from the WENSS survey which is complete to $\sim 30 \mathrm{mJy}$ (Rengelink et al. 1997). drops rapidly so that by $z=4$ the surface density is of the order of 0.001 source per square degree.

\subsection{The Radio Sky at $1400 \mathrm{MHz}$}

We calculate the source surface density and redshift distribution at $1400 \mathrm{MHz}$ to $10 \mu \mathrm{Jy}$ by transposing the $151 \mathrm{MHz}$ LRLF to $1400 \mathrm{MHz}$, and applying a beaming function as described in Jackson \& Wall (2001). We apply the spectral curvature fits (Jackson \& Wall 2001) to the rest-frame radio emission for the FRI and FRII populations. As the star-forming galaxy LRLF is determined at $1400 \mathrm{MHz}$ (Sadler et al. 2002), no transposition is necessary.

We calculate the differential source count down to $10 \mu \mathrm{Jy}$ for each of the two cosmologies; finding them to be similar we show the fit for the lambda-dominated cosmology in Figure 15.

The number of sources per square degree and the coverage fraction for each population at $1400 \mathrm{MHz}$ are given in Table 6 . We find that the total sky coverage fraction at the lowest flux density limit $(10 \mu \mathrm{Jy})$ is $0.08 \%$, i.e. there is very limited overlapping of sources on the sky.

From 1000 unique sky simulations we determine the minimum resolution which would yield an average

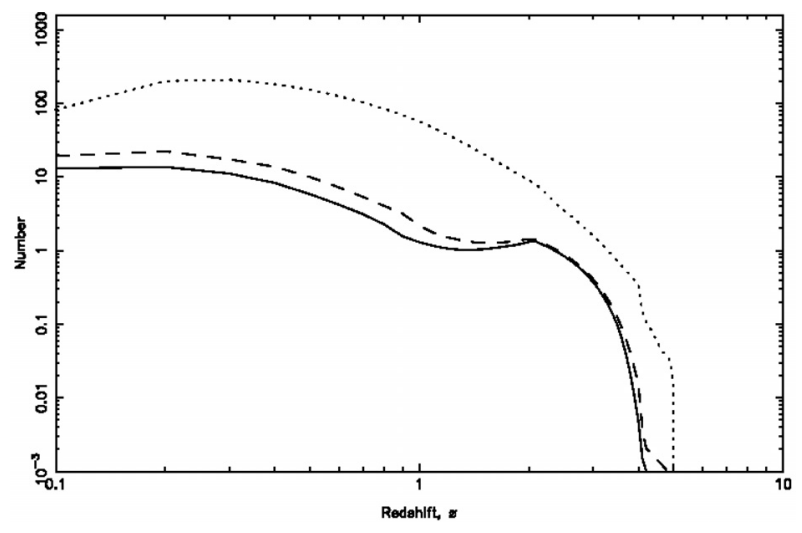

Figure 14 Predicted redshift distribution, $N(z)$, at $325 \mathrm{MHz}$ $\left(\Omega_{\mathrm{m}}=1\right)$. Number of sources per $0.1 \Delta z$ per square degree of sky for flux density limits of $2 \mathrm{mJy}$ (solid line), $1 \mathrm{mJy}$ (dashed line), and $0.1 \mathrm{mJy}$ (dotted line) derived from the fit shown in Figure 13.

Table 4. Predicted source surface densities at $325 \mathrm{MHz}$

\begin{tabular}{|c|c|c|c|c|c|c|c|c|}
\hline \multirow[t]{3}{*}{ Population } & \multicolumn{8}{|c|}{ Flux density limit } \\
\hline & \multicolumn{2}{|c|}{$5 \mathrm{mJy}$} & \multicolumn{2}{|c|}{$2 \mathrm{mJy}$} & \multicolumn{2}{|c|}{$1 \mathrm{mJy}$} & \multicolumn{2}{|c|}{$100 \mu \mathrm{Jy}$} \\
\hline & $\begin{array}{c}N \\
{\left[\mathrm{deg}^{-2}\right]}\end{array}$ & $\begin{array}{l}\text { Cover } \\
\text { fraction }\end{array}$ & $\begin{array}{c}N \\
{\left[\mathrm{deg}^{-2}\right]}\end{array}$ & $\begin{array}{l}\text { Cover } \\
\text { fraction }\end{array}$ & $\begin{array}{c}N \\
{\left[\mathrm{deg}^{-2}\right]}\end{array}$ & $\begin{array}{l}\text { Cover } \\
\text { fraction }\end{array}$ & $\begin{array}{c}N \\
{\left[\mathrm{deg}^{-2}\right]}\end{array}$ & $\begin{array}{l}\text { Cover } \\
\text { fraction }\end{array}$ \\
\hline FRI & 19 & $2 \times 10^{-3}$ & 49 & $5 \times 10^{-3}$ & 76 & $7 \times 10^{-3}$ & 527 & $3 \times 10^{-2}$ \\
\hline FRII & 17 & $6 \times 10^{-4}$ & 19 & $6 \times 10^{-4}$ & 20 & $7 \times 10^{-4}$ & 23 & $8 \times 10^{-4}$ \\
\hline Star-forming & 3 & $9 \times 10^{-6}$ & 15 & $3 \times 10^{-5}$ & 31 & $5 \times 10^{-5}$ & 985 & $6 \times 10^{-4}$ \\
\hline Total & 39 & $3 \times 10^{-2}$ & 83 & $5 \times 10^{-3}$ & 127 & $7 \times 10^{-3}$ & 1535 & $3 \times 10^{-2}$ \\
\hline Minimum resolution & \multicolumn{2}{|c|}{$19^{\prime \prime}$} & \multicolumn{2}{|c|}{$12^{\prime \prime}$} & \multicolumn{2}{|c|}{$9^{\prime \prime}$} & \multicolumn{2}{|c|}{$2^{\prime \prime}$} \\
\hline Sources overlapped & \multirow{2}{*}{\multicolumn{2}{|c|}{$\begin{array}{c}1.1 \% \\
0.0001 \%\end{array}$}} & \multirow{2}{*}{\multicolumn{2}{|c|}{$\begin{array}{c}1.8 \% \\
0.0003 \%\end{array}$}} & \multirow{2}{*}{\multicolumn{2}{|c|}{$\begin{array}{c}2.6 \% \\
0.0006 \%\end{array}$}} & \multirow{2}{*}{\multicolumn{2}{|c|}{$\begin{array}{c}9.2 \% \\
0.0164 \%\end{array}$}} \\
\hline Sky confused & & & & & & & & \\
\hline
\end{tabular}


intersource spacing of 10 primary beams on the sky. Between $S_{1400 \mathrm{MHz}}=2 \mathrm{mJy}$ and $10 \mu \mathrm{Jy}$ the minimum resolution requirement to avoid natural confusion ranges from 17 to $0.8 \operatorname{arcsec}$ (for $\Omega_{\mathrm{m}}=1$ ). Similar ranges are

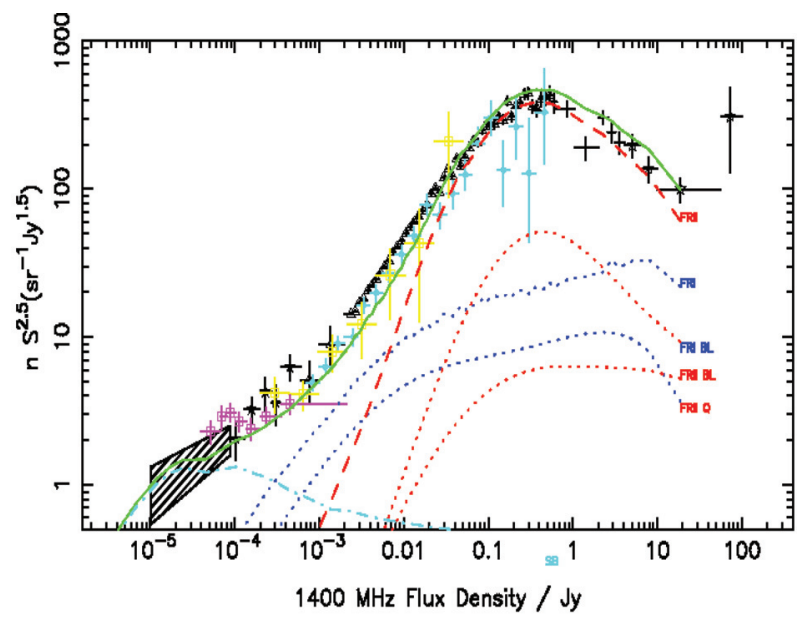

Figure 15 Normalised observed and model differential source counts at $1400 \mathrm{MHz}$ (lambda-dominated cosmology). The model count is derived from the evolving LRLF at $151 \mathrm{MHz}$ as described in the text and is comprised of three populations: FRII radio galaxies (dashed), FRI radio galaxies (dotted), and star-forming galaxies (dot-dash). Data points are from the surveys listed in Table 5. The polygon is the count estimate from the $P(D)$ (backgrounddeflection) analysis of Wall \& Cooke (1975).

Table 5. Radio source counts at $1400 \mathrm{MHz}$

\begin{tabular}{|c|c|c|c|}
\hline $\begin{array}{l}\text { Symbol in } \\
\text { Figure } 15\end{array}$ & Survey & $\begin{array}{l}\text { Flux density } \\
\text { range }\end{array}$ & Reference \\
\hline$\star$ & NRAO & $6.46-100 \mathrm{Jy}$ & Bridle et al. (1972) \\
\hline+ & NRAO & $0.705-6.46 \mathrm{Jy}$ & $\begin{array}{l}\text { Fomalont, Bridle, \& } \\
\text { Davis (1974) }\end{array}$ \\
\hline$\triangle$ & FIRST & $2.24-631 \mathrm{mJy}$ & White et al. (1997) \\
\hline$\star$ & VLA & $84 \mu \mathrm{Jy}-2.00 \mathrm{mJy}$ & $\begin{array}{l}\text { Mitchell \& Condon } \\
\text { (1985) }\end{array}$ \\
\hline$\circ$ & ATESP & $700 \mu \mathrm{Jy}-507 \mathrm{mJy}$ & $\begin{array}{l}\text { Prandoni et al. } \\
(2000)\end{array}$ \\
\hline$\square$ & Marano & $200 \mu \mathrm{Jy}-49.9 \mathrm{mJy}$ & $\begin{array}{l}\text { Gruppioni et al. } \\
\text { (1997) }\end{array}$ \\
\hline$*$ & $\mathrm{HDF}-\mathrm{N}$ & $52 \mu \mathrm{Jy}-2.1 \mathrm{mJy}$ & Richards (2000) \\
\hline
\end{tabular}

obtained for the lambda-dominated cosmology model for the reasons discussed in Section 4.3.

The power-redshift distributions at 100 and $10 \mu \mathrm{Jy}$ (Figures 16-19) show similar trends as those shown at $151 \mathrm{MHz}$ (Figures 7 and 8) with the FRI and FRII populations extending to high redshift $(z \sim 8)$.

\section{Discussion}

This paper presents a set of simulations for extragalactic radio skies which are relevant to future radio facilities where some knowledge of the surface density and redshift distribution of sources is necessary for science and/or design issues.

Our models are self-consistent with the observed radio source counts at 151,325 , and $1400 \mathrm{MHz}$. Naturally there is a high level of uncertainty in our simulations - in particular the natural confusion limits are highly dependent on the sizes of radio galaxies. We have adopted a bestguess range of intrinsic sizes to address the lack of radio source angular size data at mJy and sub-mJy flux densities.

In Table 7 we extrapolate the fits to lower flux density limits to explore the expected numbers of sources over some broad redshift ranges and for both cosmology fits. For the lambda-domindated cosmology, we find that there are of order 10-15 FRII radio sources at $z>5$ per square degree to a flux density limit of $10 \mu \mathrm{Jy}$. These high-redshift radio sources could be used to detect $\mathrm{HI} 21 \mathrm{~cm}$ absorption from the neutral intergalactic medium (Carilli, Gnedin, \& Owen 2002).

With regard to the MOST SKA prototype project, the upgraded telescope will be sensitive to point sources to $S_{325 \mathrm{MHz}} \geq 1 \mathrm{mJy}(5 \sigma \mathrm{rms})$ in a full $12 \mathrm{~h}$ synthesis. The resolution of the MOST at $325 \mathrm{MHz}$ will be $123 \times 123 \mathrm{arcsec}$ $\csc \delta$, meaning it will suffer from instrumental confusion at flux density limits below $100 \mathrm{mJy}$. Similarly at $1400 \mathrm{MHz}$ the upgraded MOST will be sensitive to point sources to $S_{1400 \mathrm{MHz}} \geq 0.2 \mathrm{mJy}$ ( $\left.5 \sigma \mathrm{rms}\right)$. From the instrumental resolution estimates of Table 6 it is obvious that the MOST will be confused at this flux density level, with source separation only possible above $5 \mathrm{mJy}$.

An exciting science goal for the upgraded MOST would be the detection of cold $(100 \mathrm{~K})$ damped Lyman- $\alpha$ systems

Table 6. Predicted source surface densities at $1400 \mathrm{MHz}$

\begin{tabular}{|c|c|c|c|c|c|c|c|c|}
\hline \multirow[t]{3}{*}{ Population } & \multicolumn{8}{|c|}{ Flux density limit } \\
\hline & \multicolumn{2}{|c|}{$2 \mathrm{mJy}$} & \multicolumn{2}{|c|}{$1 \mathrm{mJy}$} & \multicolumn{2}{|c|}{$100 \mu \mathrm{Jy}$} & \multicolumn{2}{|c|}{$10 \mu \mathrm{Jy}$} \\
\hline & $\begin{array}{c}N \\
{\left[\mathrm{deg}^{-2}\right]}\end{array}$ & $\begin{array}{l}\text { Cover } \\
\text { fraction }\end{array}$ & $\begin{array}{c}N \\
{\left[\mathrm{deg}^{-2}\right]}\end{array}$ & $\begin{array}{l}\text { Cover } \\
\text { fraction }\end{array}$ & $\begin{array}{c}N \\
{\left[\mathrm{deg}^{-2}\right]}\end{array}$ & $\begin{array}{l}\text { Cover } \\
\text { fraction }\end{array}$ & $\begin{array}{c}N \\
{\left[\mathrm{deg}^{-2}\right]}\end{array}$ & $\begin{array}{l}\text { Cover } \\
\text { fraction }\end{array}$ \\
\hline FRI & 29 & $2 \times 10^{-3}$ & 45 & $3 \times 10^{-3}$ & 366 & $1 \times 10^{-2}$ & 1913 & $6 \times 10^{-2}$ \\
\hline FRII & 17 & $5 \times 10^{-4}$ & 18 & $5 \times 10^{-4}$ & 22 & $7 \times 10^{-4}$ & 23 & $7 \times 10^{-4}$ \\
\hline Star-forming & 3 & $9 \times 10^{-6}$ & 6 & $2 \times 10^{-5}$ & 220 & $2 \times 10^{-4}$ & 5177 & $2 \times 10^{-3}$ \\
\hline Total & 49 & $2 \times 10^{-3}$ & 69 & $3 \times 10^{-3}$ & 608 & $2 \times 10^{-2}$ & 7113 & $6 \times 10^{-2}$ \\
\hline Minimum resolution & \multicolumn{2}{|c|}{$17^{\prime \prime}$} & \multicolumn{2}{|c|}{$14^{\prime \prime}$} & \multicolumn{2}{|c|}{$4^{\prime \prime}$} & \multicolumn{2}{|c|}{$0.8^{\prime \prime}$} \\
\hline Sources overlapped & \multicolumn{2}{|c|}{$0.9 \%$} & \multicolumn{2}{|c|}{$1.4 \%$} & \multicolumn{2}{|c|}{$5.6 \%$} & \multicolumn{2}{|c|}{$15.2 \%$} \\
\hline Sky confused & \multicolumn{2}{|c|}{$0 \%$} & \multicolumn{2}{|c|}{$0 \%$} & \multicolumn{2}{|c|}{$0.004 \%$} & \multicolumn{2}{|c|}{$0.079 \%$} \\
\hline
\end{tabular}




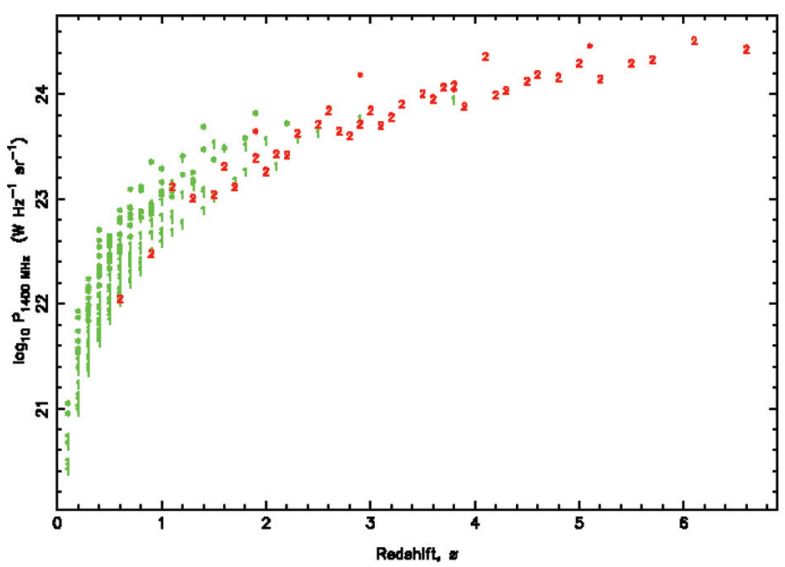

Figure 16 Power-redshift distribution, $P-z$, for FRI and FRII sources at $1400 \mathrm{MHz}$ for a flux density limit of $100 \mu \mathrm{Jy}$ derived from the fit shown in Figure 15 (lambda-dominated). Key: FRI radio galaxies (1), FRII radio galaxies (2), beamed sources $(*)$.

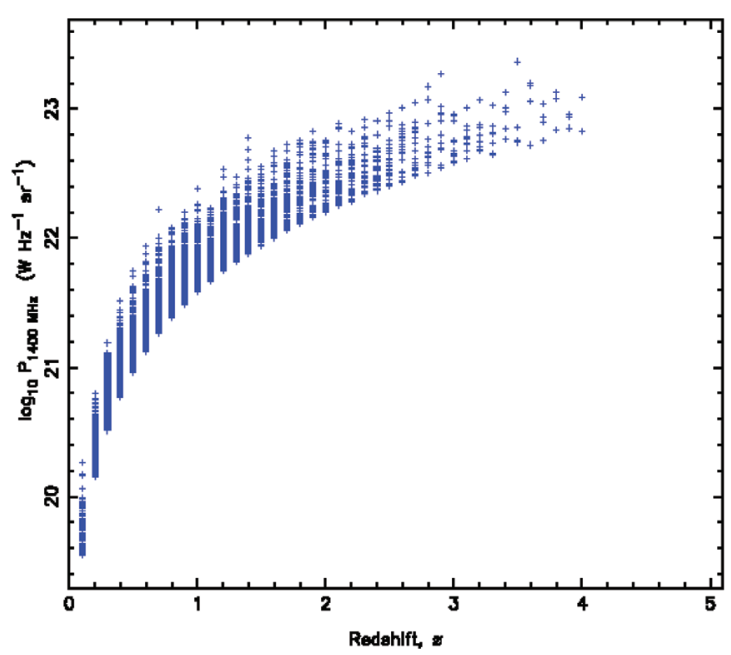

Figure 17 Power-redshift distribution, $P-z$, for star-forming galaxies (+) at $1400 \mathrm{MHz}$ for a flux density limit of $100 \mu \mathrm{Jy}$ derived from the fit shown in Figure 15 (lambda-dominated).

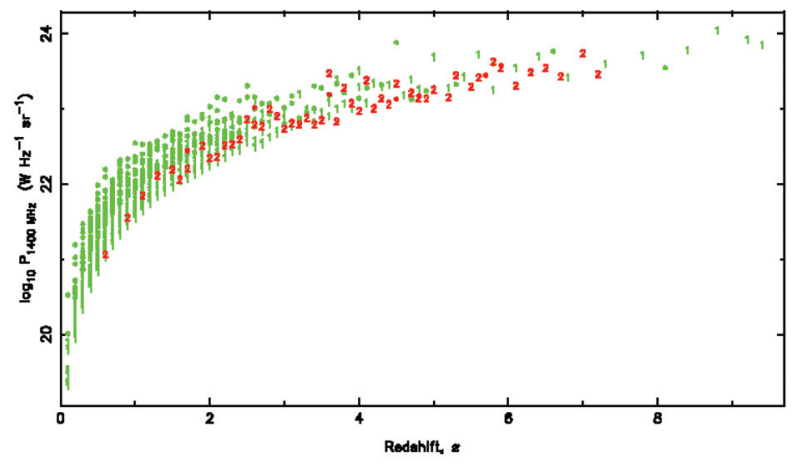

Figure 18 Power-redshift distribution, $P-z$, for FRI and FRII sources at $1400 \mathrm{MHz}$ for a flux density limit of $10 \mu \mathrm{Jy}$ derived from the fit shown in Figure 15 (lambda-dominated). Key: FRI radio galaxies (1), FRII radio galaxies (2), beamed sources (*).

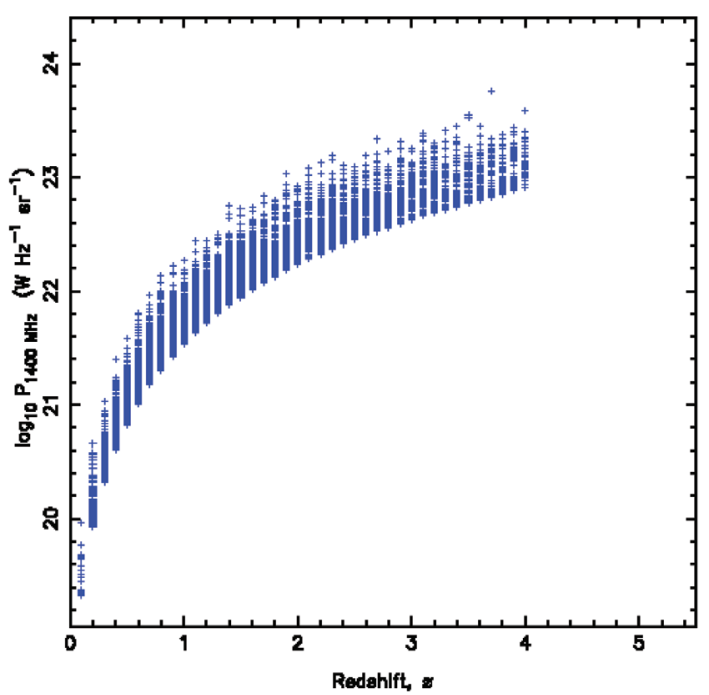

Figure 19 Power-redshift distribution, $P-z$, for star-forming galaxies (+) at $1400 \mathrm{MHz}$ for a flux density limit of $10 \mu \mathrm{Jy}$ derived from the fit shown in Figure 15 (lambda-dominated).

Table 7. Predicted numbers of sources per square degree, from the fits at $1400 \mathrm{MHz}$

\begin{tabular}{|c|c|c|c|c|c|c|c|c|c|}
\hline \multirow[t]{3}{*}{ Redshift } & \multicolumn{9}{|c|}{ Flux density limit } \\
\hline & \multicolumn{3}{|c|}{$10 \mu \mathrm{Jy}$} & \multicolumn{3}{|c|}{$1 \mu \mathrm{Jy}$} & \multicolumn{3}{|c|}{$100 \mathrm{nJy}$} \\
\hline & $N($ FRI $)$ & $N($ FRII $)$ & $N(\mathrm{SF})$ & $N($ FRI $)$ & $N($ FRII $)$ & $N(\mathrm{SF})$ & $N(\mathrm{FRI})$ & $N($ FRII $)$ & $N(\mathrm{SF})$ \\
\hline \multicolumn{10}{|l|}{$\Omega_{\mathrm{m}}=1$} \\
\hline$z<1$ & 1184 & 2 & 3577 & 3246 & 2 & 17510 & 6454 & 2 & 33918 \\
\hline$z<3$ & 1870 & 21 & 5114 & 6492 & 21 & 42454 & 16362 & 21 & 128588 \\
\hline$z<5$ & 1913 & 23 & 5177 & 7116 & 23 & 45869 & 19280 & 23 & 152492 \\
\hline$z>5$ & 0 & 0 & 0 & 0 & 0 & 0 & 0 & 0 & 0 \\
\hline \multicolumn{10}{|c|}{ Lambda-dominated } \\
\hline$z<1$ & 718 & 2 & 2556 & 1636 & 2 & 9759 & 2570 & 2 & 14238 \\
\hline$z<3$ & 1145 & 21 & 6740 & 3202 & 21 & 42865 & 6551 & 21 & 100453 \\
\hline$z<5$ & 1190 & 42 & 7584 & 3644 & 42 & 58702 & 7955 & 42 & 161671 \\
\hline$z>5$ & 17 & 13 & 0 & 384 & 14 & 0 & 2207 & 14 & 0 \\
\hline
\end{tabular}


against bright extended radio galaxies. Extended radio galaxies would provide bright background sources against which to measure the frequency and size of protogalaxies which are otherwise difficult to detect. If the upgraded MOST can achieve a $5 \sigma$ noise level at $10 \mathrm{~km} \mathrm{~s}^{-1}$ resolution of $2 \mathrm{mJy}$ in one day's observation (i.e. $9 \mathrm{~h}$ ), then damped Lyman- $\alpha$ systems could be detected against $20 \mathrm{mJy}$ radio galaxies (F. H. Briggs 2002, private communication). Our (lambda-dominated) model predicts that there will be of order 10 FRII extended radio galaxies per square degree at $S_{325 \mathrm{MHz}} \geq 20 \mathrm{mJy}$ and so this experiment should be attainable with the upgraded MOST in future years.

\section{References}

Bridle, A. H., Davis, M. M., Fomalont, E. B., \& Lequeux, J. 1972, AJ, 77, 405

Bock, D. C., Large, M. I., \& Sadler, E. M. 1999, AJ, 117, 1578

Carilli, C. L., Gnedin, N. Y., \& Owen, F. 2002, ApJ, 575, 145

Condon, J. J. 1983, ApJS, 53, 459

Condon, J. J. 1992, ARA\&A, 30, 575

Condon, J. J., Cotton, W. D., Greisen, E. W., Yin, Q. F., Perley, R. A., Taylor, G. B., \& Broderick, J. J. 1998, AJ, 115, 1693

Dunlop, J. S., \& Peacock, J. A. 1990, MNRAS, 247, 19

Fanaroff, B. L., \& Riley, J. M. 1972, MNRAS, 167, 31P

Fanti, R., Fanti, C., Schilizzi, R. T., Spencer, R. E., Rendong, N., van Breugel, W. J. M., \& Venturi, T. 1990, A\&A, 231, 333

Fomalont, E. B., Bridle, A. H., \& Davis, M. M. 1974, A\&A, 36, 273
Giallongo, E., Menci, N., Poli, F., D’Odorico, S., \& Fontana, A. 2000, ApJ, 530, L73

Gruppioni, C., Zamorani, G., de Ruiter, H. R., Parma, P., Mignoli, M., \& Lari, C. 1997, MNRAS, 286, 470

Haarsma, D. B., Partridge, R. B., Windhorst, R. A., \& Richards, E. A 2000, ApJ, 544, 641

Hales, S. E. G., Baldwin, J. E., \& Warner, P. J. 1988, MNRAS, 234, 919

Hopkins, A. M., Windhorst, R., Cram, L., \& Ekers, R. 2000, ExA, 10,419

Jackson, C. A., \& Wall, J. V. 1999, MNRAS, 304, 160

Jackson, C. A., \& Wall, J. V. 2001, in ASP Conf. Ser. 250, Particles \& Fields in Radio Galaxies, eds. R. A. Laing, \& K. M. Blundell (San Francisco, CA: ASP), 400

Laing, R. A., Riley, J. M., \& Longair, M. S. 1983, MNRAS, 204, 151

Magliocchetti, M., et al. 2002, MNRAS, 333, 100

Mitchell, K. J., \& Condon, J. J. 1985, AJ, 90, 1957

Prandoni, I., Gregorini, L., Parma, P., de Ruiter, H. R., Vettolani, G., Wieringa, M. H., \& Ekers, R. D. 2000, A\&A, 365, 392

Prandoni, I., Gregorini, L., Parma, P., de Ruiter, H. R., Vettolani, G., Zanichelli, A., \& Wieringa, M. H. 2001, A\&A, 369, 787

Rengelink, R. B., Tang, Y., de Bruyn, A. G., Miley, G. K., Bremer, M. N., Rottgering, H. J. A., \& Bremer, M. A. R. 1997, A\&AS, 124, 259

Richards, E. A. 2000, ApJ, 533, 611

Sadler, E. M., et al. 2002, MNRAS, 329, 227

Wall, J. V., \& Cooke, D. J. 1975, MNRAS, 171, 9

White, R. L., Becker, R. H., Helfand, D. J., \& Gregg, M. D. 1997, ApJ, 475, 479 


\section{Appendix A: Sky Simulations}
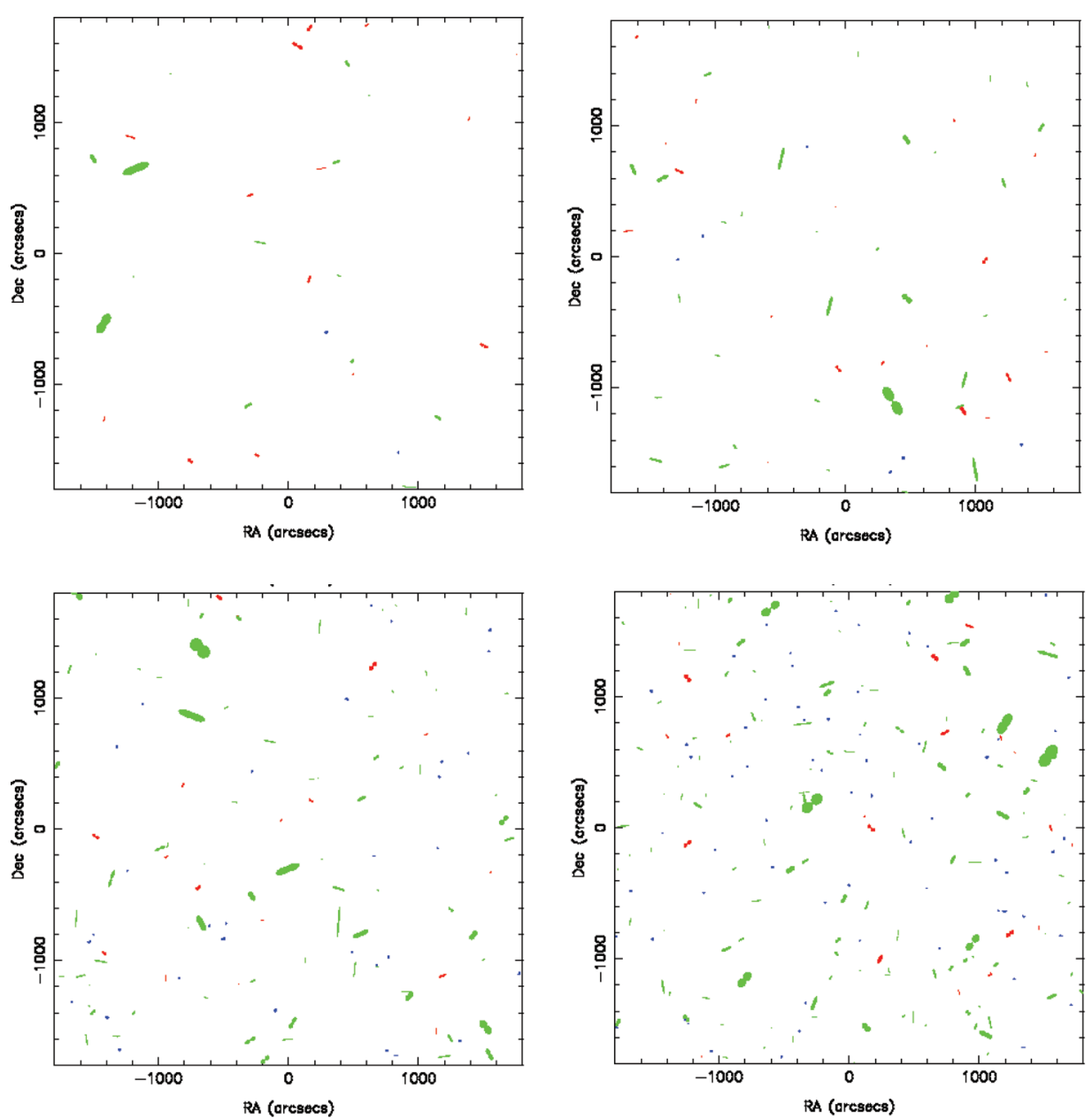

Figure 20 Simulated sky images at $151 \mathrm{MHz}$ each for a $1 \mathrm{deg}^{2}$ region. Flux density limits are 10, 5, 2, and $1 \mathrm{mJy}$ (left to right, top to bottom). There are three populations of sources shown: FRI AGN (light, double-lobed), FRII AGN (dark, double-lobed), and star-forming galaxies (dark, single-disk morphology).
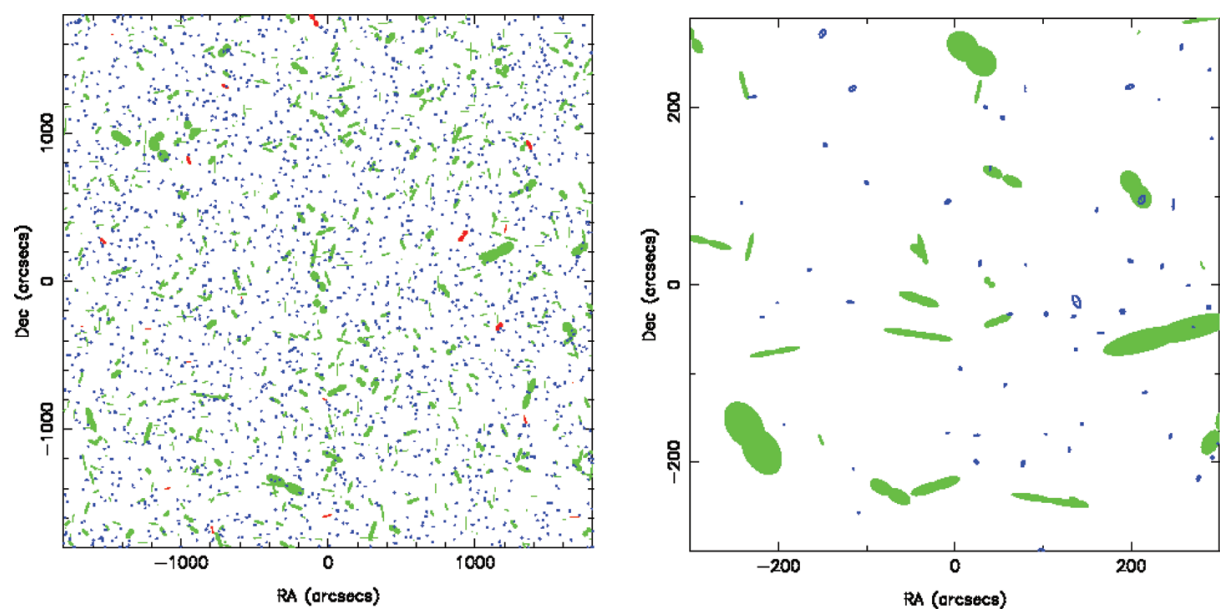

Figure 21 Simulated sky images at $151 \mathrm{MHz}$ both to a flux density limit of $100 \mu \mathrm{Jy}$. Left figure shows a region of $1 \mathrm{deg}^{2}$, right figure is $10 \operatorname{arcmin}^{2}$. There are three populations of sources shown with the same key as for Figure 20. 

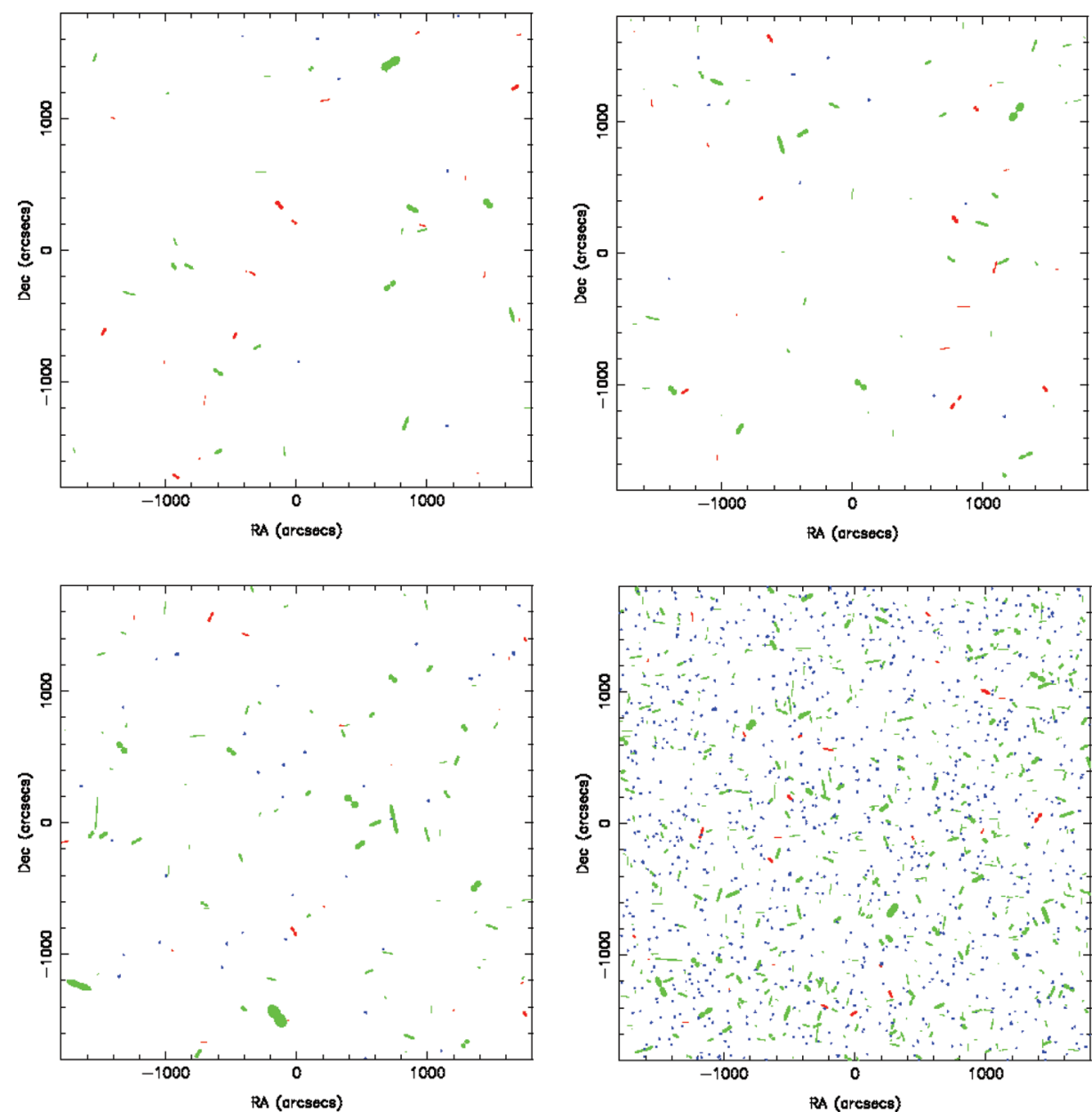

Figure 22 Simulated sky images at $325 \mathrm{MHz}$, each for a $1 \mathrm{deg}^{2}$ region. Flux density limits are 5, 2, $1 \mathrm{mJy}$, and $100 \mu \mathrm{Jy}$ (left to right, top to bottom). There are three populations of sources shown with the same key as for Figure 20.
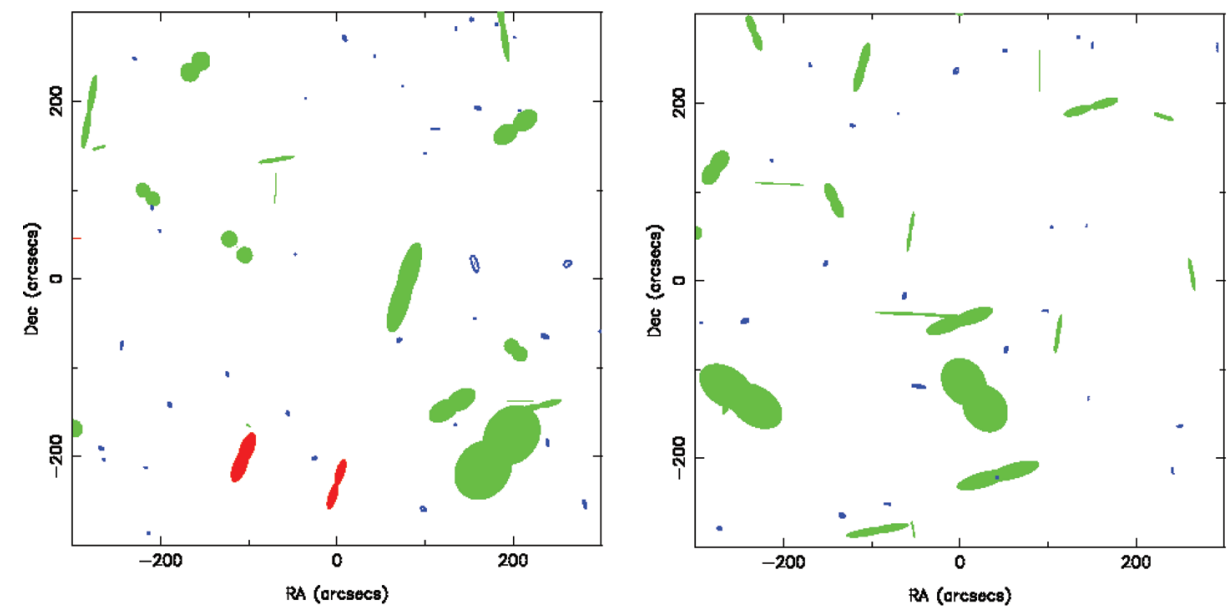

Figure 23 Simulated sky images at $325 \mathrm{MHz}$ of a $10 \mathrm{arcmin}^{2}$ region to a flux density limit of $100 \mu \mathrm{Jy}$. There are three populations of sources shown with the same key as for Figure 20. 

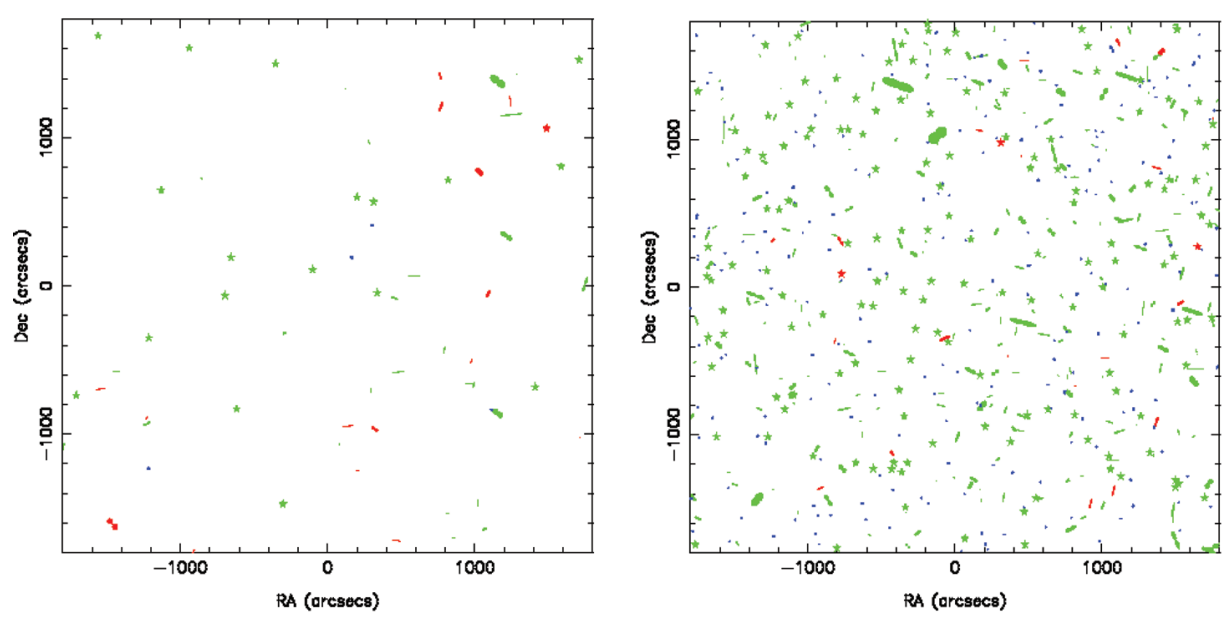

Figure 24 Simulated sky images at $1400 \mathrm{MHz}$, each for a $1 \mathrm{deg}^{2}$ region. Flux density limits are $1 \mathrm{mJy}$ and $100 \mu \mathrm{Jy}$ (left, right). There is a total of five populations of sources, with the same key as for Figure 20, plus the additional beamed FRIIs (dark, star), and FRIs (light, star).
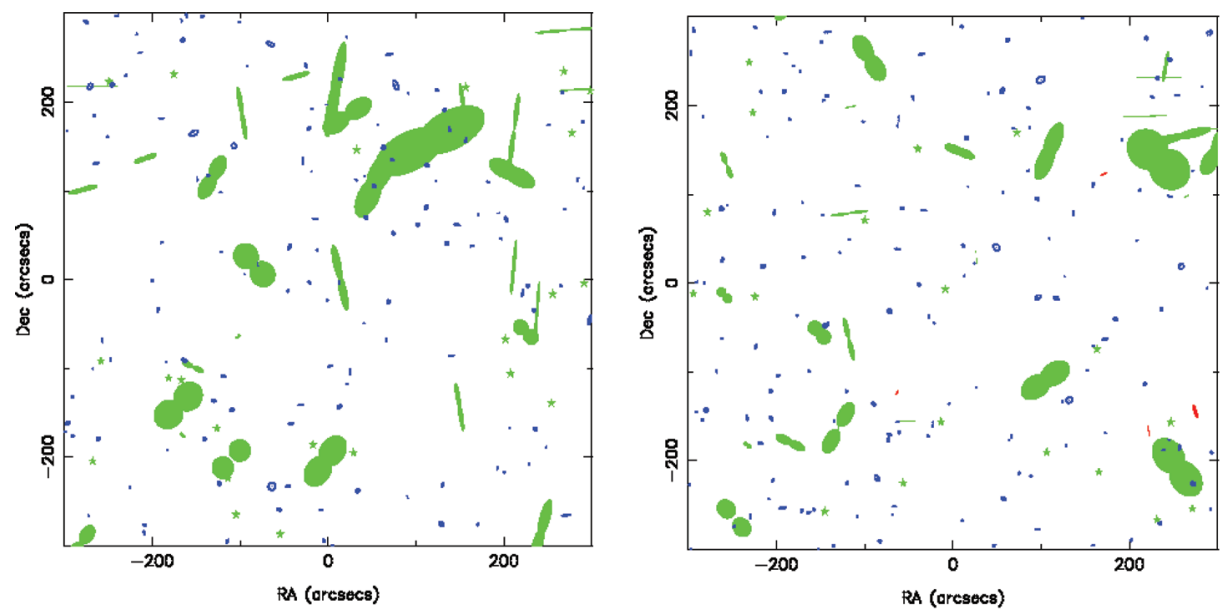

Figure 25 Simulated sky images at $1400 \mathrm{MHz}$, each of a $10 \mathrm{arcmin}^{2}$ region and with a flux density limit of $10 \mu \mathrm{Jy}$. There are three populations of sources shown with the same key as for Figure 24. 